\title{
Static black holes with a negative cosmological constant: Deformed horizon and anti-de Sitter boundaries
}

\author{
Hirotaka Yoshino $*$ Tohru Ohba $\oplus$ and Akira Tomimatsu \\ Department of Physics, Graduate School of Science, \\ Nagoya University, Chikusa, Nagoya 464-8602, Japan
}

(Dated: November 27, 2018)

\begin{abstract}
Using perturbative techniques, we investigate the existence and properties of a new static solution for the Einstein equation with a negative cosmological constant, which we call the deformed black hole. We derive a solution for a static and axisymmetric perturbation of the Schwarzschild-anti-de Sitter black hole that is regular in the range from the horizon to spacelike infinity. The key result is that this perturbation simultaneously deforms the two boundary surfaces-i.e., both the horizon and spacelike two-surface at infinity. Then we discuss the Abbott-Deser mass and the AshtekarMagnon one for the deformed black hole, and according to the Ashtekar-Magnon definition, we construct the thermodynamic first law of the deformed black hole. The first law has a correction term which can be interpreted as the work term that is necessary for the deformation of the boundary surfaces. Because the work term is negative, the horizon area of the deformed black hole becomes larger than that of the Schwarzschild-anti-de Sitter black hole, if compared under the same mass, indicating that the quasistatic deformation of the Schwarzschild-anti-de Sitter black hole may be compatible with the thermodynamic second law (i.e., the area theorem).
\end{abstract}

PACS numbers: $04.20 .-\mathrm{q}, 04.70 . \mathrm{Bw}, 04.70 . \mathrm{Dy}, 04.20 . \mathrm{Cv}$

\footnotetext{
*Electronic address: hyoshino@allegro.phys.nagoya-u.ac.jp

$\dagger$ Electronic address: tohba@allegro.phys.nagoya-u.ac.jp

${ }_{\ddagger}^{\ddagger}$ Electronic address: atomi@allegro.phys.nagoya-u.ac.jp
}

Typeset by REVTEX 1 


\section{INTRODUCTION}

Recently, spacetimes with a negative cosmological constant $\Lambda$ have attracted a lot of attention in various contexts, such as the AdS/conformal field theory (CFT) correspondence (see 1] for a recent review) or the Randall-Sundrum brane world scenario [2]. One of the necessary investigations in these contexts would be to analyze the classical feature of the spacetime with negative $\Lambda$, such as the black hole physics in these spacetimes.

Black hole physics in spacetimes with $\Lambda<0$ has a remarkable feature: the spatial topology of the black hole horizon is not necessarily spherical even in stationary four-dimensional spacetimes. In the 1990s, new solutions which represent the black holes with nonspherical topology horizons were discovered [3, 4, 5, 6, 17, 8, 9, 10, 11] in addition to the well-known solutions: i.e., the Schwarzschild-anti-de Sitter black hole and the Kerr-anti-de Sitter black hole. For example, Lemos constructed a static solution of an infinitely large planar black hole for the four-dimensional spacetime with $\Lambda<0$ [3]. He also pointed out that the planar solution also represents the cylindrical black hole or the toruslike black hole by appropriate compactifications of this spacetime. By these procedures, the topology of the black hole horizon becomes the cylinder $\left(R \times S^{1}\right)$ or the torus $\left(S^{1} \times S^{1}\right)$, and conformal spacelike infinity has the same topology as the horizon. Lemos generalized his static cylindrical black hole solution to the rotating one [4]. The generalization including charge and dilaton can be found in [5, 6, 7]. There exists another solution of the black hole with unusual topology. Åminneborg et al. constructed a solution of a black hole whose horizon is a Riemann twosurface and can take an arbitrary genus value $g>1$ by appropriate compactifications [8]. The topology of the conformal spacelike infinity is the same as that of the horizon due to this compactification. The generalization for the charged case can be found in [9, 10], and rotating topological black holes were introduced by Klemm et al. [11].

Readers might wonder why the black hole solutions with such various kinds of horizon topology exist even in four dimensions. The black hole topology theorem proved by Hawking claims that the topology of the horizon is $S^{2}$ [12]. But the assumptions of this theorem are that the spacetime is asymptotically flat and that certain energy conditions hold, which are both incompatible with the negative cosmological constant. In the presence of negative $\Lambda$, the black hole physics becomes far richer than the case of $\Lambda=0$. On the other hand, there are some theorems that restrict black hole solutions in spacetimes with $\Lambda<0$. Anderson et 
al. proved that the Schwarzschild-anti-de Sitter black hole is the unique static solution for asymptotically anti-de Sitter vacuum spacetimes with $\Lambda<0$ [13]. Galloway et al. proved some theorems that restrict the black hole solutions in $\Lambda<0$ spacetimes [14]. However, we expect that there would be a great possibility of the existence of unknown solution series.

In this paper, we consider the existence of the series of black hole solutions which describes a continuous change from the Schwarzschild-anti-de Sitter black hole to the cylindrical or planar one. Our expectation is easily understood by looking at the recent study of black strings and black holes in higher-dimensional Kaluza-Klein spacetimes. Gregory and Laflamme analyzed the stability of the black string in higher-dimensional spacetime [15]. They showed that the black string is unstable for a perturbation along the string, if the wavelength is sufficiently large. It was also shown that there is a static perturbation, which was a strong implication for the existence of a new sequence of static solutions. This static perturbation was investigated in detail by Gubser [16], and subseqently Wiseman [17] and Kudoh and Wiseman [18] numerically solved the sequence that connects the black string to the black hole in higher-dimensional Kaluza-Klein spacetime. We can expect that a similar situation would occur in four-dimensional $\Lambda<0$ spacetimes.

Motivated by this expectation, we analyze the static perturbation of the Schwarzschild-anti-de Sitter spacetime as a starting point. The existence of such solutions is the manifestation of the existence of the new solution series. We consider the axisymmetric, even-parity perturbations in the Regge-Wheeler formalism [19]. Each component of the metric perturbation is represented by a product of a radial function and Legendre's polynomial $P_{l}(\cos \theta)$. In the case of $\Lambda=0$, the solution diverges either at the horizon or at infinity [19]. If $\Lambda<0$, however, it is possible to construct a solution which does not diverge everywhere from the horizon to infinity for all multipole modes corresponding to $l=2,3, \ldots$. The horizon of the perturbed solution is not geometrically spherical. Hence we call this solution a deformed black hole hereafter. Because some radial functions of the perturbation components asymptote to constant values at spacelike infinity, our solution is not asymptotically anti-de Sitter: a two-surface at spacelike infinity is also deformed. This is consistent with the uniqueness theorem of Anderson et al. However, our solution still describes the weakly asymptotically anti-de Sitter spacetime, in the sense of the AshtekarMagnon definition [20]. Our result also does not contradict the theorems derived by Galloway et al. 
To understand the physical implication of the black hole deformation, we would like to discuss some features of this solution such as the mass, the horizon area, and the first law of black hole thermodynamics. Because some of the metric coefficients diverge at infinity and to take a limit to infinity is a delicate problem, there are ambiguities in defining the mass of the spacetime with $\Lambda<0$. Several mass definitions in the asymptotically anti-de Sitter spacetimes have been proposed (see [21] and references therein), and some of them are applicable also to the weakly asymptotically anti-de Sitter spacetimes. Among these, we use two well-known mass definitions proposed by Abbott and Deser [22] and by Ashtekar and Magnon [20], which can be easily applied to deformed black holes. The first-order perturbation deforms the Schwarzschild-anti-de Sitter black hole without changing the two masses and the horizon area. Hence we should consider the $l=0$ mode of the second-order perturbation, which is generated by the terms of a product of two first-order perturbation components in the second-order equation. The two definitions give totally different results for the mass derived from the second-order perturbation. The Abbott-Deser mass diverges to minus infinity, while the Ashtekar-Magnon mass gives a finite value. As we will discuss in detail later, this result would be due to the fact that the Abbott-Deser mass is not gauge invariant at second order. Since the definition of the Ashtekar-Magnon mass is covariant, it would provide a real amount of energy contained in the spacetime with a deformed black hole. Therefore we expect that quasistatic deformation of the Schwarzschild-anti-de Sitter black hole occurs with a finite change in the total energy and thus an investigation of the thermodynamic first law of the deformed black holes with this mass definition is meaningful.

One can easily find that the Schwarzschild-anti-de Sitter black holes obey the thermodynamic laws like asymptotically flat black holes. The first law was extended to the static black holes with unusual topology by Vanzo [23]. He used the mass defined as the on-shell value of the Hamiltonian, which gives the same value as the Abbott-Deser mass, and showed that the first law holds with this mass definition. It was explicitly shown that the first law with the usual form also holds for the Kerr-Newman-anti-de Sitter black holes [24], and there are some approaches to the proof of the first law for the asymptotically anti-de Sitter, stationary black hole spacetimes under more general assumptions [25, 26]. We will analyze the first law of the deformed black holes as follows. There are two characteristic scales for these spacetimes: i.e., the Schwarzschild radius $R_{S} \equiv 2 m$ and the anti-de Sitter radius $R_{A} \equiv \sqrt{-3 / \Lambda}$. We analytically consider the case that the Schwarzschild radius is 
much smaller than the anti-de Sitter radius (i.e., $\alpha \equiv R_{S} / R_{A} \ll 1$ ) and the case that the Schwarzschild radius is much larger than the anti-de Sitter radius (i.e., $\alpha \gg 1$ ). Then we numerically solve the $\alpha \sim 1$ cases. We will explicitly construct the solutions for the first-order perturbation and calculate the horizon area and the Ashtekar-Magnon mass. Our calculation shows that the first law in the usual form approximately holds for the $\alpha \gg 1$ case, and it does not hold for the other cases. In other words, the first law has a correction term which can be interpreted as the work term necessary for the deformation of the two boundary surfaces of the spacetime: i.e., the horizon and two-surface at spacelike infinity. If we compare the horizon area of the Schwarzschild-anti-de Sitter black hole and that of the deformed black hole under the same mass, the latter becomes larger. Therefore deformation of the Schwarzschild-anti-de Sitter black hole will be claimed to be a process compatible with the usual area law.

The outline of the paper is as follows. In Sec. II, we derive the equations for a static, axisymmetric perturbation of Schwarzschild-anti-de Sitter spacetime. We show that there exists a solution for the first-order perturbation which does not diverge everywhere, and that this spacetime is the weakly asymptotically anti-de Sitter spacetime. Then we derive the second-order equation for the $l=0$ mode and the general formula for the horizon area of the deformed black hole. In Sec. III, we derive the general formulas of the Abbott-Deser mass and the Ashtekar-Magnon mass for the deformed black holes. We discuss the property of these two definitions and show that the Abbott-Deser mass is not gauge invariant. In Sec. IV, we explicitly construct the solutions for the first-order perturbation in two cases $\alpha \ll 1$ and $\alpha \gg 1$. Then we analyze the first law of the deformed black holes. In Sec. V, we numerically calculate for the $\alpha \sim 1$ case and discuss the dependence of the first law on the value of $\alpha$. In Sec. VI, we summarize our results and discuss their physical implications.

\section{STATIC, AXISYMMETRIC PERTURBATION}

In this section, we derive the static, axisymmetric perturbation of the Schwarzschild-antide Sitter black hole. The background metric is given in the Schwartzschild-like coordinates $(t, r, \theta, \phi)$ as follows:

$$
d \hat{s}^{2}=-e^{2 \nu_{0}} d t^{2}+e^{2 \mu_{0}} d r^{2}+r^{2}\left(d \theta^{2}+\sin ^{2} \theta d \phi^{2}\right)
$$




$$
e^{2 \nu_{0}}=e^{-2 \mu_{0}}=1-\frac{2 m}{r}-\frac{1}{3} \Lambda r^{2} .
$$

As we see from the investigations in [19] and [27], the metric of a spherically symmetric spacetime with first- and second-order even-parity static perturbations can be written in diagonal form

$$
d \hat{s}^{2}=-e^{2 \nu} d t^{2}+e^{2 \mu} d r^{2}+e^{2 \psi} r^{2}\left(d \theta^{2}+\sin ^{2} \theta d \phi^{2}\right)
$$

where $\nu, \mu$, and $\psi$ are expanded with a small deformation parameter $\epsilon$ as follows:

$$
\begin{aligned}
& \nu=\nu_{0}+\epsilon \nu_{1}+\epsilon^{2} \nu_{2}, \\
& \mu=\mu_{0}+\epsilon \mu_{1}+\epsilon^{2} \mu_{2}, \\
& \psi=\epsilon \psi_{1}+\epsilon^{2} \psi_{2} .
\end{aligned}
$$

Here all first- and second- order functions are given by the sum of the products of a radial function and Legendre's polynomial $P_{l}(\cos \theta)$, after the appropriate gauge transformations. We use this Regge-Wheeler-like gauge for an investigation of the deformed black holes.

\section{A. First-order perturbation}

We give the first-order perturbation as

$$
\begin{aligned}
& \nu_{1}=-\mu_{1}=-H^{(1)}(r) P_{l}(\cos \theta), \\
& \psi_{1}=K^{(1)}(r) P_{l}(\cos \theta),
\end{aligned}
$$

where the first equality in Eq. (17) is imposed by the difference of $\theta \theta, \phi \phi$ components of the Einstein equation $R_{\mu \nu}=\Lambda g_{\mu \nu}$. We restrict our attention to $l \geq 2$, because $l=0$ and $l=1$ modes are absorbed to the coordinate transformation and the changing of the mass. The first-order equations become

$$
\begin{gathered}
r^{2} e^{2 \nu_{0}} H_{, r r}^{(1)}+2 r\left(r e^{2 \nu_{0}}\right)_{, r} H_{, r}^{(1)}-r^{2}\left(e^{2 \nu_{0}}\right)_{, r} K_{, r}^{(1)}-\left[2 \Lambda r^{2}+l(l+1)\right] H^{(1)}=0, \\
r^{2} e^{2 \nu_{0}}\left(H_{, r r}^{(1)}-2 K_{, r r}^{(1)}\right)+2 r\left(r e^{2 \nu_{0}}\right)_{, r} H_{, r}^{(1)} \\
-r^{2}\left[\left(e^{2 \nu_{0}}\right)_{, r}+4 r^{-1} e^{2 \nu_{0}}\right] K_{, r}^{(1)}-\left[2 \Lambda r^{2}-l(l+1)\right] H^{(1)}=0, \\
e^{2 \nu_{0}}\left(H_{, r}^{(1)}-K_{, r}^{(1)}\right)+\left(e^{2 \nu_{0}}\right)_{, r} H^{(1)}=0,
\end{gathered}
$$




$$
\begin{aligned}
r^{2} e^{2 \nu_{0}} K_{, r r}^{(1)}-2 r e^{2 \nu_{0}} H_{, r}^{(1)}+r^{2}\left[\left(e^{2 \nu_{0}}\right)_{, r}+4 r^{-1} e^{2 \nu_{0}}\right] K_{, r}^{(1)} & \\
& -2\left(r e^{2 \nu_{0}}\right)_{, r} H^{(1)}-\left(l^{2}+l-2\right) K^{(1)}=0,
\end{aligned}
$$

where, $r$ denotes the derivative with respect to $r$. These equations come from $t t, r r, r \theta$ components and the sum of the $\theta \theta, \phi \phi$ components of the Einstein equation, respectively. Although there are four equations for two functions $H^{(1)}$ and $K^{(1)}$, these equations do not overdetermine $H^{(1)}$ and $K^{(1)}$ because we can derive Eq. (9) and the derivative of Eq. (12) using Eq. (10), Eq. (11), and their derivatives. Eliminating $K^{(1)}$ from Eqs. (91) and (11), we obtain the equation for the quantity $M \equiv r^{2} e^{2 \nu_{0}} H^{(1)}$ :

$$
M_{, r r}-2\left(r^{-1}+\nu_{0, r}\right) M_{, r}-e^{-2 \nu_{0}}\left[2 \Lambda+r^{-2}\left(l^{2}+l-2\right)\right] M=0 .
$$

Eliminating $K_{, r r}^{(1)}$ and $K_{, r}^{(1)}$ from Eqs. (91), (10), (11), and (12), we find that $K^{(1)}$ is expressed in terms of $H^{(1)}$ as

$$
K^{(1)}=\frac{1}{\left(l^{2}+l-2\right)}\left\{r^{2}\left(e^{2 \nu_{0}}\right)_{, r} H_{, r}^{(1)}+\left[r^{2}\left(2 \nu_{0, r}\right)\left(e^{2 \nu_{0}}\right)_{, r}+l^{2}+l-2 e^{2 \nu_{0}}\right] H^{(1)}\right\} .
$$

Now we show the existence of solutions which do not diverge in the range from the horizon to spatial infinity. The two independent solutions for $H^{(1)}$ of Eq. (13) are $\left(r-r_{h}\right)$ and $1 /\left(r-r_{h}\right)$ in the neighborhood of the horizon, while $1 / r$ and $1 / r^{2}$ for large $r$. Here we have introduced the horizon radius $r_{h}$, which is the maximum solution of $e^{2 \nu_{0}}\left(r_{h}\right)=0$. Thus, if we choose $\left(r-r_{h}\right)$ near the horizon, the solution $H^{(1)}$ becomes regular everywhere because it behaves like $\sim 1 / r$ at large $r$. The behavior of $H^{(1)}$ at infinity can be written as

$$
H^{(1)}=\frac{a_{1}}{r}+\frac{a_{2}}{r^{2}}+\frac{a_{3}}{r^{3}}+\cdots,
$$

where $a_{3}, a_{4}, \ldots$ are expressed with $a_{1}$ and $a_{2}$ like

$$
\begin{aligned}
& a_{3}=-\frac{3\left(l^{2}+l-4\right)}{2 \Lambda} a_{1}, \\
& a_{4}=-\frac{1}{2 \Lambda}\left[18 m a_{1}+\left(l^{2}+l-6\right) a_{2}\right],
\end{aligned}
$$

because $H^{(1)}$ contains only two integral constants. The ratio of $a_{1}$ and $a_{2}$ is determined by imposing the regularity at the horizon. Using Eq. (14), we see that the behavior of $K^{(1)}$ at infinity becomes

$$
K^{(1)}=c_{0}+\frac{c_{1}}{r}+\frac{c_{2}}{r^{2}}+\cdots,
$$


where the coefficients can be determined using Eq. (14) as follows:

$$
\begin{aligned}
& c_{0}=\frac{2 \Lambda a_{2}}{3\left(l^{2}+l-2\right)}, \\
& c_{1}=-a_{1}, \\
& c_{2}=0 .
\end{aligned}
$$

Thus, $K^{(1)}$ is also finite at $r=\infty$. Similarly, if we write the behavior of $H^{(1)}$ near the horizon as

$$
H^{(1)}=\tilde{a}_{1}\left(r-r_{h}\right)+\tilde{a}_{2}\left(r-r_{h}\right)^{2}+\cdots
$$

the behavior of $K^{(1)}$ near the horizon becomes

$$
K^{(1)}=\tilde{c}_{0}+\tilde{c}_{1}\left(r-r_{h}\right)+\cdots,
$$

where the coefficients can be determined using Eq. (14) such as

$$
\begin{aligned}
& \tilde{c}_{0}=\frac{4\left(3 m-r_{h}\right) \tilde{a}_{1}}{l^{2}+l-2}, \\
& \tilde{c}_{1}=2 \tilde{a}_{1} .
\end{aligned}
$$

Thus two functions $H^{(1)}$ and $K^{(1)}$ remain finite everywhere from the horizon to infinity, and hence we have established the existence of the new solution series of $\Lambda<0$ spacetimes.

Now we examine some properties of this solution. The induced metric on a two-surface $t=$ const and $r=r_{h}$ becomes

$$
d \hat{s}^{2}=\left[1+2 \epsilon \tilde{c}_{0} P_{l}(\cos \theta)\right] r_{h}^{2}\left(d \theta^{2}+\sin ^{2} \theta d \phi^{2}\right),
$$

which implies that the geometry of the horizon in (say) the $l=2$ case is prolate for $\tilde{c}_{0}>0$ and oblate for $\tilde{c}_{0}<0$. Thus the horizon geometry deviates from the geometrically spherical surface and this solution represents the deformed black hole. To see the structure of spacelike infinity, we consider a conformal transformation $d s^{2}=\Omega^{2} d \hat{s}^{2}$ where we choose $\Omega=r^{-1}$. The induced metric of $r=$ const surface at infinity of the conformally transformed spacetime becomes

$$
d s^{2}=\frac{1}{3} \Lambda d t^{2}+\left[1+2 \epsilon c_{0} P_{l}(\cos \theta)\right]\left(d \theta^{2}+\sin ^{2} \theta d \phi^{2}\right),
$$

which means that the conformal boundary of this spacetime is also deformed. This indicates that this spacetime is not asymptotically anti-de Sitter in the sense of the Ashtekar-Magnon 
definition [20]. According to their definition, the spacetime is asymptotically anti-de Sitter if the spacetime satisfies the Einstein equation with $\Lambda<0$ and with an energy momentum tensor that satisfies an appropriate falloff condition, and its conformal boundary $\mathcal{I}$ is topologically $S^{2} \times R$, and the conformal group of $\mathcal{I}$ is the anti-de Sitter group. The last condition is equivalent to that $\mathcal{I}$ admits a global chart $(t, \theta, \phi)$ such that the metric on $\mathcal{I}$ is conformally related to the metric

$$
d s_{0}^{2}=\frac{1}{3} \Lambda d t^{2}+d \theta^{2}+\sin ^{2} \theta d \phi^{2}
$$

This is clearly inconsistent with Eq. (27). However, the spacetime of the deformed black hole is weakly asymptotically anti-de Sitter [20]: this is defined by the above conditions except the last condition. The deformed black hole spacetime has a deformed surface at spacelike infinity, and thus the existence of this solution is not contradictory to the uniqueness theorem of the asymptotically anti-de Sitter black hole [13].

To solve analytically $H^{(1)}$ and $K^{(1)}$ for general $\alpha$ is rather difficult. We will explicitly construct the analytic solutions in two simple cases $\alpha \equiv 2 m \sqrt{-\Lambda / 3} \ll 1$ and $\alpha \gg 1$ in Sec. IV and the numerical solutions in the $\alpha \sim 1$ cases in Sec. V. Here, we show that $H^{(1)}$ and $K^{(1)}$ do not change their sign for $r_{h}<r<\infty$. Using Eq. (11), $K^{(1)}$ is given by

$$
K^{(1)}=H^{(1)}+\int_{r_{h}}^{r} 2 \nu_{0, r} H^{(1)} d r+\tilde{c}_{0}
$$

We consider the $\tilde{a}_{1}>0$ case (hence $\tilde{c}_{0}>0$ ). If $H^{(1)}$ changes its sign from positive to negative at $r=r_{c}, K^{(1)}\left(r_{c}\right)$ should be positive from Eq. (29) and $H_{, r}^{(1)}\left(r_{c}\right)$ should be negative. However, this contradicts Eq. (14). Hence $H^{(1)}$ is always positive. Using Eq. (29) again, we see that $K^{(1)}$ also always takes a positive value. The proof for the case $\tilde{a}_{1}<0$ is similar. Thus $H^{(1)}$ and $K^{(1)}$ have the same sign and do not change their sign. This means that there is a natural relation between the geometry of the horizon and that of the conformal boundary of this spacetime. Because $\tilde{c}_{0}$ and $c_{0}$ have the same sign, the conformal boundary is prolate if the horizon is prolate and vice versa. Moreover, using Eq. (29) again, we see that $\left|K^{(1)}-H^{(1)}\right|$ is a monotonically increasing function of $r$. Because $H^{(1)}\left(r_{h}\right)=H^{(1)}(\infty)=0$, we find $\left|K^{(1)}\left(r_{h}\right)\right|<\left|K^{(1)}(\infty)\right|$ and hence $\left|\tilde{c}_{0}\right|<\left|c_{0}\right|$. Thus the conformal boundary at infinity is more deformed compared to the horizon.

As we see from Eq. (26), the first-order perturbation does not change the area of the horizon. It also does not affect the mass of the spacetime, as we will see in the next section. 
Hence to see the change in the mass and horizon area due to the deformation of the black hole, we should consider the $l=0$ mode of the second-order perturbation.

\section{B. $\quad l=0$ mode of second-order perturbation}

We consider the second-order perturbation which is generated by the $l \geq 2$ mode of the first-order perturbation of the black hole. The second-order perturbation is given as follows:

$$
\begin{aligned}
& \nu_{2}=-H_{0}^{(2)}(r)+\sum_{n \neq 0} H_{n}^{(2)}(r) P_{n}(\cos \theta), \\
& \mu_{2}=\tilde{H}_{0}^{(2)}(r)+\sum_{n \neq 0} \tilde{H}_{n}^{(2)}(r) P_{n}(\cos \theta), \\
& \psi_{2}=K_{0}^{(2)}(r)+\sum_{n \neq 0} K_{n}^{(2)}(r) P_{n}(\cos \theta) .
\end{aligned}
$$

Because there remains the degree of freedom to choose the $r$ coordinate, we can impose $H^{(2)} \equiv H_{0}^{(2)}=\tilde{H}_{0}^{(2)}$ only for the $l=0$ mode. We will treat only the functions of the $l=0$ mode, $H^{(2)}$ and $K^{(2)} \equiv K_{0}^{(2)}$, for the second-order perturbation.

The second-order Einstein equations of the $l=0$ mode are

$$
\begin{aligned}
& r^{2} e^{2 \nu_{0}} H_{, r r}^{(2)}+2 r\left(r e^{2 \nu_{0}}\right)_{, r} H_{, r}^{(2)}-r^{2}\left(e^{2 \nu_{0}}\right)_{, r} K_{, r}^{(2)}-2 \Lambda r^{2} H^{(2)} \\
& =\frac{2}{2 l+1}\left\{e^{2 \nu_{0}} r^{2}\left[\left(H_{, r}^{(1)}\right)^{2}-H_{, r}^{(1)} K_{, r}^{(1)}\right]+\left[\Lambda r^{2}+l(l+1)\right]\left(H^{(1)}\right)^{2}-l(l+1) H^{(1)} K^{(1)}\right\}
\end{aligned}
$$

$$
\begin{array}{r}
r^{2} e^{2 \nu_{0}}\left(H_{, r r}^{(2)}-2 K_{, r r}^{(2)}\right)+2 r\left(r e^{2 \nu_{0}}\right)_{, r} H_{, r}^{(2)}-r^{2}\left[\left(e^{2 \nu_{0}}\right)_{, r}+4 r^{-1} e^{2 \nu_{0}}\right] K_{, r}^{(2)}-2 \Lambda r^{2} H^{(2)} \\
=\frac{2}{2 l+1}\left\{e^{2 \nu_{0}} r^{2}\left[\left(H_{, r}^{(1)}\right)^{2}+\left(K_{, r}^{(1)}\right)^{2}-H_{, r}^{(1)} K_{, r}^{(1)}\right]+\left[\Lambda r^{2}-l(l+1)\right]\left(H^{(1)}\right)^{2}\right. \\
\left.+l(l+1) H^{(1)} K^{(1)}\right\},
\end{array}
$$

$$
\begin{array}{r}
r^{2} e^{2 \nu_{0}} K_{, r r}^{(2)}-2 r e^{2 \nu_{0}} H_{, r}^{(2)}+r^{2}\left[\left(e^{2 \nu_{0}}\right)_{, r}+4 r^{-1} e^{2 \nu_{0}}\right] K_{, r}^{(2)}-2\left(r e^{2 \nu_{0}}\right)_{, r} H^{(2)}+2 K^{(2)} \\
=\frac{-1}{2 l+1}\left\{2 r^{2} e^{2 \nu_{0}}\left[\left(K_{, r}^{(1)}\right)^{2}-H_{, r}^{(1)} K_{, r}^{(1)}\right]+\left(2 \Lambda r^{2}+l^{2}+l-2\right)\left(H^{(1)}\right)^{2}\right. \\
\left.-2\left(l^{2}+l-2\right) H^{(1)} K^{(1)}+2\left(l^{2}+l-1\right)\left(K^{(1)}\right)^{2}\right\},
\end{array}
$$


where we have used the formulas

$$
\begin{aligned}
\left\langle\left[P_{l}(\cos \theta)\right]^{2}\right\rangle & =1 /(2 l+1), \\
\left\langle\left[d P_{l}(\cos \theta) / d \theta\right]^{2}\right\rangle & =l(l+1) /(2 l+1),
\end{aligned}
$$

where

$$
\langle f\rangle \equiv \frac{1}{2} \int_{0}^{\pi} f(\theta) \sin \theta d \theta .
$$

These equations come from $t$, $r r$ components and the sum of the $\theta \theta, \phi \phi$ components of the Einstein equation, respectively. The $r \theta$ component and the difference of the $\theta \theta, \phi \phi$ components provide no conditions for the $l=0$ mode. Similarly to the case of the firstorder perturbation, these equations do not overdetermine $H^{(2)}$ and $K^{(2)}$.

The homogeneous solution for Eqs. (33), (34), and (35) is given by

$$
\begin{aligned}
& K_{\text {hom }}^{(2)}=C_{1}+C_{2} / r \\
& H_{\text {hom }}^{(2)}=e^{-2 \nu_{0}}\left[C_{1}-\left(e^{2 \nu_{0}}\right)_{, r} C_{2} / 2+C_{3} / r\right] .
\end{aligned}
$$

This is not physical because these perturbations are absorbed to the coordinate transformation and changing the mass like

$$
\begin{aligned}
& \bar{t}=\left(1-\epsilon^{2} C_{1}\right) t, \\
& \bar{r}=\left(1+\epsilon^{2} C_{1}\right) r+\epsilon^{2} C_{2}, \\
& \bar{m}=\left(1+3 \epsilon^{2} C_{1}\right) m+\epsilon^{2} C_{3} .
\end{aligned}
$$

Moreover, $H_{h o m}^{(1)}$ diverges on the horizon. Hence, in order to obtain a solution that does not diverge for $r_{h} \leq r \leq \infty$, we should impose the homogeneous solution to be zero. This means that $K^{(2)}$ behaves like

$$
K^{(2)}=\frac{d_{2}}{r^{2}}+\frac{d_{3}}{r^{3}}+\cdots,
$$

for large $r$, where $d_{2}, d_{3}, \ldots$ are determined like

$$
\begin{aligned}
& d_{2}=-\frac{a_{1}^{2}}{2(2 l+1)} \\
& d_{3}=\frac{l(l+1) a_{1} c_{0}}{(2 l+1) \Lambda} .
\end{aligned}
$$

The fact that $K^{(2)}=O\left(1 / r^{2}\right)$ at large $r$ provides a boundary condition for solving $K^{(2)}$ for $r_{h}<r<\infty$. The solution is written as

$$
K^{(2)}=\frac{-1}{(2 l+1)} \int_{r}^{\infty} \frac{1}{r^{\prime \prime 2}} \int_{r^{\prime \prime}}^{\infty}\left[r^{\prime 2}\left(K_{, r^{\prime}}^{(1)}\right)^{2}-\frac{2 l(l+1)}{e^{2 \nu_{0}}} H^{(1)}\left(H^{(1)}-K^{(1)}\right)\right] d r^{\prime} d r^{\prime \prime} .
$$


We also see that $H^{(2)}$ behaves like

$$
H^{(2)}=\frac{b_{2}}{r^{2}}+\frac{b_{3}}{r^{3}}+\cdots .
$$

Although $b_{2}$ is determined like

$$
b_{2}=\frac{1}{2(2 l+1)}\left(a_{1}^{2}-\frac{6 c_{0}^{2}}{\Lambda}\left(l^{2}+l-1\right)\right),
$$

it is not possible to determine $b_{3}$ in terms of $a_{1}$ and $c_{0}$, because we cannot impose $C_{3}$ of the homogeneous solution to be zero at infinity. It is determined only by explicitly solving $H^{(2)}$ with an appropriate boundary condition at the horizon $r=r_{h}$ which we introduce later.

Near the horizon, the behavior of $K^{(2)}$ is written as

$$
K^{(2)}=\tilde{d}_{0}+\tilde{d}_{1}\left(r-r_{h}\right)+\cdots
$$

where the coefficients are determined only by explicitly calculating Eq. (47):

$$
\begin{aligned}
& \tilde{d}_{0}=\frac{1}{(2 l+1)}\left(-\frac{c_{0} a_{1}}{r_{h}}+\frac{1}{2}\left(c_{0}^{2}-\tilde{c}_{0}^{2}\right)+\int_{r_{h}}^{\infty} \frac{\left(r-r_{h}\right) l(l+1)}{r_{h} r e^{2 \nu_{0}}} H^{(1)}\left(2 H^{(1)}-K^{(1)}\right) d r\right), \\
& \tilde{d}_{1}=\frac{1}{(2 l+1)}\left(\frac{c_{0} a_{1}}{r_{h}^{2}}-2 \tilde{c}_{0} \tilde{a}_{1}-\int_{r_{h}}^{\infty} \frac{l(l+1)}{r_{h}^{2} e^{2 \nu_{0}}} H^{(1)}\left(2 H^{(1)}-K^{(1)}\right) d r\right) .
\end{aligned}
$$

Once $K^{(2)}$ is calculated, we find that $H^{(2)}$ behaves like

$$
H^{(2)}=\tilde{b}_{0}+\tilde{b_{1}}\left(r-r_{h}\right)+\cdots
$$

near the horizon, where the coefficients are determined by Eqs. (35) and (34) like

$$
\begin{aligned}
& \tilde{b}_{0}=\frac{r_{h}}{2\left(3 m-r_{h}\right)}\left(\left(3 m-r_{h}\right) \tilde{d}_{1}+\tilde{d}_{0}+\frac{l^{2}+l-1}{2 l+1} \tilde{c}_{0}^{2}\right), \\
& \tilde{b}_{1}=\frac{1}{2} \tilde{d}_{1}+\frac{\Lambda r_{h}^{2}}{2\left(3 m-r_{h}\right)} \tilde{b}_{0} .
\end{aligned}
$$

The fact $H^{(2)}\left(r_{h}\right)=\tilde{b}_{0}$ gives a boundary condition for solving $H^{(2)}$ for $r_{h} \leq r \leq \infty$. The solution of $H^{(2)}$ satisfying this boundary condition is written as

$$
\begin{array}{r}
H^{(2)}=\frac{1}{2(2 l+1) r e^{2 \nu_{0}}} \int_{r_{h}}^{r}\left\{(2 l+1)\left[2 K^{(2)}+\left(r^{\prime 2} e^{2 \nu_{0}}\right)_{, r^{\prime}} K_{, r^{\prime}}^{(2)}\right]-{r^{\prime}}^{2} e^{2 \nu_{0}} K_{, r^{\prime}}^{(1)}\left(2 H_{, r^{\prime}}^{(1)}-K_{, r^{\prime}}^{(1)}\right)\right. \\
\left.+\left(2 \Lambda r^{\prime 2}+3 l^{2}+3 l-2\right)\left(H^{(1)}\right)^{2}-2\left(l^{2}+l-1\right) K^{(1)}\left(2 H^{(1)}-K^{(1)}\right)\right\} d r^{\prime} . \quad(56)
\end{array}
$$

Now we calculate the horizon area of the deformed black hole. The induced metric on the horizon $t=$ const and $r=r_{h}$ becomes

$$
d \hat{s}^{2}=\left.\left(1+2 \psi_{1}+2 \psi_{1}^{2}+2 \psi_{2}\right)\right|_{r=r_{h}} r_{h}^{2}\left(d \theta^{2}+\sin ^{2} \theta d \phi^{2}\right)
$$


Substituting Eqs. (8) and (32) and then using Eqs. (23) and (150), the change in the horizon area $\delta A$ is easily calculated:

$$
\frac{\delta A}{A}=2 \epsilon^{2}\left(\frac{\tilde{c}_{0}^{2}}{2 l+1}+\tilde{d}_{0}\right)
$$

where $A \equiv \pi r_{h}^{2}$ is the area of the Schwarzschild-anti-de Sitter black hole. Because $\tilde{d}_{0}$ is given in Eq. (51), we can calculate $\delta A$ without explicitly constructing solutions of $H^{(2)}$ and $K^{(2)}$. This quantity $\delta A / A$ provides an indicator for the strength of nonlinear effect near the horizon.

We also consider the area of the $t=$ const surface of the conformal boundary $\mathcal{I}$ in the case where we choose the conformal factor $\Omega=r^{-1}$, because it becomes the measure for the strength of nonlinear effect near infinity. We write this area and the increase in this area $S$ and $\delta S$, respectively. This is given by

$$
\frac{\delta S}{S}=2 \epsilon^{2}\left(\frac{c_{0}^{2}}{2 l+1}\right)
$$

Because this is proportional to $c_{0}^{2}$ and is positive definite, this quantity provides a natural factor for normalizing the thermodynamical quantities, such as $\delta A / A$ and $\delta m / m$.

\section{MASS OF THE DEFORMED BLACK HOLE}

In this section, we calculate the mass of the deformed black hole. As we mentioned in the Introduction, we consider two definitions of mass: the Abbott-Deser mass [22] and the Ashtekar-Magnon mass [20]. We will find that these two definitions of mass give totally different results and will discuss the reason for this difference.

\section{A. Abbott-Deser mass}

Abbott and Deser found a conserved quantity for the spacetimes with a cosmological constant [22]. They divided the metric tensor $\hat{g}_{\mu \nu}$ into two parts:

$$
\hat{g}_{\mu \nu}=\bar{g}_{\mu \nu}+h_{\mu \nu}
$$

where $\bar{g}_{\mu \nu}$ is the solution of the Einstein equation which has the Killing vector field $\bar{\xi}_{\mu}$, and $h_{\mu \nu}$ represents a deviation from the background metric $\bar{g}_{\mu \nu}$. The conserved quantity 
associated with the Killing vector field $\bar{\xi}_{\mu}$ is

$$
E(\bar{\xi})=\frac{1}{8 \pi} \int \sqrt{-\bar{g}} d^{2} x\left(\bar{\xi}_{\nu} D_{\beta} K^{\mu \alpha \nu \beta}-K^{\mu \beta \nu \alpha} D_{\beta} \bar{\xi}_{\nu}\right) D_{\mu} t D_{\alpha} r
$$

where $\bar{g}$ is the determinant of $\bar{g}_{\mu \nu}, D_{\mu}$ denotes the covariant derivative with respect to the background metric $\bar{g}_{\mu \nu}, t$ and $r$ are the usual time and radial coordinates, and $K^{\mu \alpha \nu \beta}$ is the superpotential defined by

$$
K^{\mu \alpha \nu \beta}=\frac{1}{2}\left(\bar{g}^{\mu \beta} H^{\nu \alpha}+\bar{g}^{\nu \alpha} H^{\mu \beta}-\bar{g}^{\mu \nu} H^{\alpha \beta}-\bar{g}^{\alpha \beta} H^{\mu \nu}\right)
$$

where

$$
H^{\mu \nu}=h^{\mu \nu}-\bar{g}^{\mu \nu} h_{\alpha}^{\alpha} / 2 .
$$

The indices are moving with respect to $\bar{g}_{\mu \nu}$, and the integral is taken on the two-sphere at infinity $r=\infty$. If $\bar{\xi}_{\mu}$ is the usual (past-directed) timelike Killing vector, $E(\bar{\xi})$ becomes the total mass generated by $h_{\mu \nu}$ which is called the Abbott-Deser mass $M_{A D}$.

Now we calculate the Abbott-Deser mass of the deformed black hole. We adopt the Schwarzschild-anti-de Sitter spacetime given by Eqs. (11) and (2) as the background spacetime. By a straightforward calculation, we find that the Abbott-Deser mass of the spacetime of Eq. (3) becomes

$$
M_{A D}=\lim _{r \rightarrow \infty} \frac{r^{2}}{8 \pi} \int\left[e^{2 \nu_{0}}\left(\frac{1}{r}\left(e^{2\left(\mu-\mu_{0}\right)}-e^{2 \psi}\right)-\left(e^{2 \psi}\right)_{, r}\right)+\frac{1}{2}\left(e^{2 \nu_{0}}\right)_{, r}\left(e^{2 \psi}-1\right)\right] d \Omega,
$$

where $d \Omega \equiv \sin \theta d \theta d \phi$ and we used $\bar{\xi}^{\mu}=(-1,0,0,0)$. By expanding this formula in $\epsilon$ using Eqs. (5) and (6), $M_{A D}$ is written like

$$
M_{A D}=\epsilon M_{A D}^{(1)}+\epsilon^{2} M_{A D}^{(2)}+\cdots .
$$

Because $\mu_{1}$ and $\psi_{1}$ are proportional to $P_{l}(\cos \theta)$, the integration in Eq. (64) immediately leads to $M_{A D}^{(1)}=0$ for $l \geq 2$. For the second-order Abbott-Deser mass $M_{A D}^{(2)}$, we find, by substituting Eqs. (71), (8), (31), and (32) and then using Eqs. (15), (18), (44), and (48),

$$
M_{A D}^{(2)}=\lim _{r \rightarrow \infty}\left(\frac{2 \Lambda c_{0} a_{1}}{3(2 l+1)} r^{2}+\cdots\right)=-\infty .
$$

Thus the Abbott-Deser mass diverges to minus infinity at second order.

One interpretation for this divergence of the Abbott-Deser mass is that the deformed black hole is a far-lower-energy state compared to the Schwarzschild-anti-de Sitter black hole and 
the Schwarzschild-anti-de Sitter black hole would rapidly deform toward the lower-energy state. However, we would like to point out that such an interpretation may not be correct. To see this, we consider the coordinate transformation $r \rightarrow r+\epsilon a$ of the Schwarzschild-anti-de Sitter metric. The resulting metric is given by Eq. (3) with

$$
\begin{aligned}
& e^{-2 \nu}=e^{2 \mu}=e^{2 \mu_{0}}+\left(e^{2 \mu_{0}}\right)_{, r} a \epsilon+\left(e^{2 \mu_{0}}\right)_{, r r} a^{2} \epsilon^{2} / 2+\cdots, \\
& e^{2 \psi}=1+2 a \epsilon / r+a^{2} \epsilon^{2} / r^{2} .
\end{aligned}
$$

Substituting these formulas to Eq. (64), we find $M_{A D}^{(1)}=0$ and

$$
M_{A D}^{(2)}=\lim _{r \rightarrow \infty}\left(-\frac{5 \Lambda a^{2}}{6} r+\cdots\right)=\infty .
$$

Thus the Abbott-Deser mass for the perturbation generated by the coordinate transformation also diverges at second order. This indicates that the Abbott-Deser mass is not gauge invariant at second order and hence its divergence may be spurious. In fact, we can obtain a finite mass for the deformed black hole, if we use the covariant definition of mass given by Ashtekar and Magnon.

\section{B. Ashtekar-Magnon mass}

Ashtekar and Magnon constructed a conserved quantity of a weakly asymptotically anti-de Sitter spacetime $\left(\hat{M}, \hat{g}_{\mu \nu}\right)[20]$. They considered the conformally transformed spacetime $\left(M, g_{\mu \nu}\right)$ where $g_{\mu \nu}=\Omega^{2} \hat{g}_{\mu \nu}$ and the situation that $M$ has a boundary $\mathcal{I}$ whose topology is $R \times S^{2}$. Their conserved quantity is defined on the spacelike $S^{2}$ surface (denoted by $\mathcal{C}$ ) on the conformal boundary $\mathcal{I}$ as follows:

$$
Q_{\xi} \equiv-\frac{1}{8 \pi}\left(-\frac{3}{\Lambda}\right)^{3 / 2} \int_{\mathcal{C}} \Omega^{-1} C_{\alpha \mu \beta \nu} \xi^{\alpha} n^{\mu} N^{\beta} n^{\nu} d S
$$

where $n_{\mu}=\nabla_{\mu} \Omega, N^{\beta}$ is the timelike unit normal on $\mathcal{C}, \xi^{\alpha}$ is the conformal Killing vector field on $\mathcal{I}, d S$ is the proper area element of $\mathcal{C}$, and $C_{\alpha \mu \beta \nu}$ denotes the Weyl tensor of $M$. All index moving and covariant derivatives are with respect to the metric of the conformally transformed spacetime $\left(M, g_{\mu \nu}\right)$. This definition is conformally invariant with the same choice of the coordinate components of $\xi^{\mu}$. If $\xi^{\mu}$ is the (future-directed) timelike Killing vector field, this conserved quantity is the Ashtekar-Magnon mass $M_{A M}$ of the spacetime. 
For the axisymmetric spacetime with metric (3), we find by a straightforward calculation that the Ashtekar-Magnon mass becomes

$$
M_{A M}=\lim _{r \rightarrow \infty}-\sqrt{\frac{-3}{16 \Lambda}} \int_{0}^{\pi} \xi^{t} r^{2} e^{\nu}\left(e^{-2 \mu}\left[\nu_{, r r}+\nu_{, r}\left(\nu_{, r}-\mu_{, r}\right)\right]+\frac{e^{-2 \psi}}{r^{2}} \mu_{, \theta} \nu_{, \theta}+\frac{\Lambda}{3}\right) e^{2 \psi} \sin \theta d \theta .
$$

If we calculate this quantity for the Schwarzschild-anti-de Sitter spacetime, we obtain $M_{A M}=m$ choosing $\xi^{t}=1$. We can easily show that this quantity does not change under the coordinate transformation $r \rightarrow r+\epsilon a$.

In the above definition, how to choose the norm of $\xi^{\mu}$ has not been specified. Here we would like to discuss this criterion using one concrete example, because the mass calculation of the deformed black hole crucially depends on the choice of the norm of $\xi^{\mu}$. If we make a coordinate transformation $r \rightarrow a r$ and $t \rightarrow b t$ to the Schwarzschild-anti-de Sitter spacetime, the resulting metric becomes Eq. (3) with

$$
\begin{aligned}
b^{-2} e^{2 \nu}=a^{2} e^{-2 \mu} & =1-2 m / a r-\Lambda a^{2} r^{2} / 3 \\
e^{2 \psi} & =a^{2} .
\end{aligned}
$$

Choosing $\Omega=1 / r$, the metric of the conformal boundary $\mathcal{I}$ becomes

$$
d s^{2}=\left(\Lambda a^{2} b^{2} / 3\right) d t^{2}+a^{2}\left(d \theta^{2}+\sin ^{2} \theta d \phi^{2}\right)
$$

Calculating Eq. (71) for this metric, we obtain $M_{A M}=\xi^{t} b m$. Because the mass should be invariant under the coordinate transformation, this means that we should choose $\xi^{t}=b^{-1}$. One of the natural general criteria that recover this choice is as follows: if there is a conformal transformation such that the norm of the Killing vector field $\xi^{\mu}$ becomes constant on $\mathcal{I}$, we should choose $\xi^{\mu}$ that satisfy

$$
(3 / \Lambda) \xi^{\mu} \xi_{\mu}=S / 4 \pi
$$

for the calculation of the Ashtekar-Magnon mass, where $S$ denotes the proper area of the $t=$ const surface on $\mathcal{I}$. We adopt this criterion in calculating the Ashtekar-Magnon mass of the deformed black hole.

Now we calculate the Ashtekar-Magnon mass of the deformed black hole. From the above discussion, we use $\xi^{t}$ in the calculation of Eq. (171) as follows:

$$
\xi^{t}=\lim _{r \rightarrow \infty} \sqrt{\left\langle e^{2 \psi}\right\rangle}=\lim _{r \rightarrow \infty} 1+\epsilon^{2}\left(\left\langle\psi_{2}\right\rangle+\left\langle\psi_{1}^{2}\right\rangle\right)+O\left(\epsilon^{4}\right)
$$


where the definition of $\langle f\rangle$ is given in Eq. (38). Substituting this formula and expanding Eq. (71) using Eqs. (41), (15), and (6), $M_{A M}$ can be written like

$$
M_{A M}=M_{A M}^{(0)}+\epsilon M_{A M}^{(1)}+\epsilon^{2} M_{A M}^{(2)}+\cdots .
$$

We immediately obtain $M_{A M}^{(0)}=m$ and $M_{A M}^{(1)}=0$ for $l \geq 2$. Substituting Eqs. (77), (86), (30), (31), and (32) and then using Eqs. (15), (18), (44), and (48) for $M_{A M}^{(2)}$, we obtain, after a rather lengthy calculation,

$$
M_{A M}^{(2)}=\frac{1}{3} \Lambda\left(\frac{2\left(a_{1} a_{2}-a_{3} c_{0}\right)}{2 l+1}-b_{3}\right)+\frac{2 a_{1} c_{0}}{2 l+1}+\frac{3 m c_{0}^{2}}{2 l+1} .
$$

This is finite in contrast to the Abbott-Deser mass. Because the Ashtekar-Magnon mass is a covariant definition of mass which is welldefined even in the weakly asymptotically anti-de Sitter spacetimes, we can regard it as a real amount of energy contained in the spactimes with deformed black holes. The validity of the Ashtekar-Magnon mass is also supported by the expectation that the continuous change of mass should result from the continuous deformed black hole series. Hence we consider that there is a possibility of the quasistatic deformation of the spacetime boundaries in weakly asymptotically anti-de Sitter spacetimes and we investigate the thermodynamic law of the deformed black holes with this mass definition in the following two sections.

To simplify Eq. (178), we consider the method of expressing $b_{3}$ with $H^{(1)}$ and $K^{(1)}$. By summing Eqs. (33) and (134) and then rewriting the right-hand side using Eqs. (91) and (11), we find

$$
\begin{aligned}
\left(r^{2} e^{2 \nu_{0}} H_{, r}^{(2)}\right)_{, r}+2 m H_{, r}^{(2)}-\frac{2}{3} \Lambda\left(r^{3} H^{(2)}\right)_{, r}-\left(r^{2} e^{2 \nu_{0}} K_{, r}^{(2)}\right)_{, r} & \\
= & \frac{1}{2 l+1}\left[\left(r^{2} e^{2 \nu_{0}} H^{(1)} H_{, r}^{(1)}\right)_{, r}-l(l+1)\left(H^{(1)}\right)^{2}\right] .
\end{aligned}
$$

Integrating this equation from the horizon to large $r$ and substituting Eqs. (15), (44), and (48), we obtain

$$
\frac{\Lambda}{3} b_{3}=\frac{5 l(l+1)-6}{2(2 l+1)} a_{1} c_{0}+2\left(3 m-r_{h}\right) \tilde{b}_{0}-\frac{l(l+1)}{2 l+1} \int_{r_{h}}^{\infty}\left(H^{(1)}\right)^{2} d r .
$$

Substituting this formula, Eqs. (16) and (19) into Eq. (78), we finally find

$$
M_{A M}^{(2)}=\frac{-\left(l^{2}+l+2\right)}{2(2 l+1)} a_{1} c_{0}-2\left(3 m-r_{h}\right) \tilde{b}_{0}+\frac{l(l+1)}{2 l+1} \int_{r_{h}}^{\infty}\left(H^{(1)}\right)^{2} d r+\frac{3 m c_{0}^{2}}{2 l+1},
$$

where $\tilde{b}_{0}$ can be written in terms of $\tilde{d}_{0}$ and $\tilde{d}_{1}$ using Eq. (54), which in turn are given in Eqs. (51) and (52). Hence we can express $M_{A M}^{(2)}$ in terms of the quantities of only the first-order perturbation. 
IV. APPROXIMATE CALCULATION FOR $\alpha \gg 1$ AND $\alpha \ll 1$

As we mentioned in the Secs. I and II, to solve analytically the equations of the perturbation is rather difficult. However, we can construct the solution approximately in the two simple cases: one is the case that the anti-de Sitter radius is much larger than the Schwarzschild radius $\alpha \ll 1$, and the other is that the anti-de Sitter radius is much smaller than the Schwarzschild radius $\alpha \gg 1$. In this section, we will show the solution for these two cases and calculate the horizon area and the Ashtekar-Magnon mass. Using these results, we can discuss the properties of the first law of the black hole thermodynamics for the deformed black holes.

\section{A. $\alpha \ll 1$ case}

Setting a new coordinate $x \equiv r / 2 m$, the function $e^{2 \nu_{0}}$ becomes

$$
e^{2 \nu_{0}}=1-1 / x+(\alpha x)^{2}
$$

In the case of $\alpha \ll 1$, the horizon location is $x=x_{h} \simeq 1$. In the region $1 \leq x \lesssim \alpha^{-2 / 3}$, the order of the second term is larger than $O\left(\alpha^{2 / 3}\right)$ and the order of the third term is smaller than $O\left(\alpha^{2 / 3}\right)$. Hence the sum of the first and second terms is much larger than the third term: the spacetime is Schwarzshild-like. In the region $\alpha^{-2 / 3} \lesssim x \leq \infty$, the order of the second term is smaller than $O\left(\alpha^{2 / 3}\right)$ and the order of the third term is greater than $O\left(\alpha^{2 / 3}\right)$. Because the sum of the first and third terms is much greater than the second term, the spacetime is similar to the anti-de Sitter spacetime. Hence, we can use the matching method: we construct solutions for the Schwarzschild spacetime and the anti-de Sitter spacetime in the regions $1 \leq x \lesssim \alpha^{-2 / 3}$ and $\alpha^{-2 / 3} \lesssim x \leq \infty$, respectively, and then match these two solutions in the overlapping region $x \sim \alpha^{-2 / 3}$.

The general solutions of $H^{(1)}$ for Schwarzschild spacetime are given in [19]. The solutions that satisfy the boundary condition on the horizon can be expressed in terms of associated Legendre's polynomials, $H_{i n}^{(1)}=P_{l}^{2}(2 x-1)$. To construct a solution $H^{(1)}$ in the anti-de Sitter regime, we introduce a new coordinate $y \equiv \sqrt{-\Lambda / 3} r$. Setting $Y \equiv y^{2}$ and $M=Y^{(l+2) / 2} \tilde{M}$, Eq. (13) in the anti-de Sitter regime becomes

$$
Y(1+Y) \tilde{M}_{, Y Y}+[(l+1 / 2) Y+(l+3 / 2)] \tilde{M}_{, Y}+[l(l-1) / 4] \tilde{M}=0 .
$$


Because this equation is related to the hypergeometric equation, one of the solutions becomes $\tilde{M}={ }_{2} F_{1}(l / 2,(l-1) / 2 ; l+3 / 2 ;-Y)$, and the corresponding solution is

$$
H_{\text {out }}^{(1)}=\frac{y^{l}}{y^{2}+1}{ }_{2} F_{1}\left(l / 2,(l-1) / 2 ; l+3 / 2 ;-y^{2}\right),
$$

which behaves like $H_{\text {out }}^{(1)} \simeq y^{l}$ in the matching region. Since the behavior of $H_{\text {in }}^{(1)}$ is proportional to $x^{l}$ in the overlapping region, we can match these two solutions. The solution of $H_{\text {in }}^{(1)}$ that smoothly continues to Eq. (84) can be written as

$$
H_{i n}^{(1)}=-\left(\frac{\alpha}{2}\right)^{l} \frac{(l-2) !}{(2 l-1) ! !} P_{l}^{2}(2 x-1) .
$$

Although the convergence region of Eq. (184) is $0 \leq y \leq 1$, we can make an analytic continuation to the region $1 \leq y \leq \infty$ using well-known techniques. Hence we have constructed the solution of $H^{(1)}$ in the $\alpha \ll 1$ case. Using Eq. (14), we can also write down the solutions $K_{\text {in }}^{(1)}$ and $K_{\text {out }}^{(1)}$ in terms of associated Legendre's polynomials and the hypergeometric functions, respectively, although we do not show them explicitly here. Using this solution of $K^{(1)}$ or observing the behavior of $H_{i n}^{(1)}$ and $H_{\text {out }}^{(1)}$ near the horizon and infinity and then using Eqs. (19) and (24), we find

$$
\frac{\tilde{c}_{0}}{c_{0}}=\frac{\Gamma((l-1) / 2) \Gamma((l+3) / 2) \Gamma(l+3)}{4 \Gamma(l+3 / 2) \Gamma(l+1 / 2)}\left(\frac{\alpha}{4}\right)^{l} .
$$

Hence, for $\alpha \ll 1$, the deformation of the horizon is much smaller than that of spacelike infinity, and this tendency is enhanced for larger $l$.

Now that we have constructed a solution of the first-order perturbation, we would like to calculate the mass and horizon area. However, this calculation for general $l$ requires integration of the products of the hypergeometric functions, and the analytic calculation is rather difficult. Hence we consider analytically only the $l=2$ case, where the function in Eq. (84) reduces to the elementary functions. For $l>2$, we numerically evaluate the relation of the area and mass. Before doing this, we would like to remark on the general properties of the second-order solution in the $\alpha \ll 1$ case. As we can see from Eqs. (47) and (56), the order of the second-order perturbation becomes $O\left(\alpha^{-1} \epsilon^{2}\right)$, which is much larger than $\epsilon^{2}$. This indicates that the nonlinear effect rapidly increases with an increase in $\epsilon$. This perturbative analysis is only reliable for a sufficiently small $\epsilon$ that satisfies $\epsilon<\alpha$.

Now we calculate the horizon area and the mass for the $l=2$ case. The solutions of $H_{\text {out }}^{(1)}$ 
and $K_{\text {out }}^{(1)}$ corresponding to Eq. (184) can be rewritten as

$$
\begin{aligned}
H_{\text {out }}^{(1)} & =\frac{5}{8 y^{3}\left(y^{2}+1\right)}\left[-y\left(5 y^{2}+3\right)+3\left(y^{2}+1\right)^{2} \arctan y\right], \\
K_{\text {out }}^{(1)} & =\frac{5}{2}-\frac{15}{8 y^{3}}\left[y+\left(y^{2}-1\right) \arctan y\right] .
\end{aligned}
$$

Using Eqs. (58), (59), and (81), we find

$$
\begin{aligned}
\frac{\delta A}{A} & \simeq \frac{3 \pi}{8}(12 \log 2-11) \alpha^{-1} \epsilon^{2}, \\
\frac{\delta m}{m} & \simeq \frac{3 \pi}{4}(3 \log 2-4) \alpha^{-1} \epsilon^{2}, \\
\frac{\delta S}{S} & \simeq \frac{5}{2} \epsilon^{2},
\end{aligned}
$$

where $\delta m \equiv M_{A M}^{(2)} \epsilon^{2}$. Note that both $\delta A$ and $\delta m$ are negative. Because $(\delta A / A) /(\delta S / S)=$ $O\left(\alpha^{-1}\right)$, the back reaction to the horizon area is far larger than that to the spacelike area at infinity. This result implies

$$
\frac{(\delta m / \delta A)}{(\kappa / 8 \pi)}=\frac{12 \log 2-16}{12 \log 2-11} \simeq 2.86,
$$

which indicates that the first law in the usual form does not hold for deformed black holes. In other words, the first law for the deformation of the Schwarzschild-anti-de Sitter black holes has a correction term such that

$$
\delta m=\frac{\kappa}{8 \pi} \delta A+\delta W
$$

where $\delta W$ can be expressed in terms of the coefficients of $H^{(1)}$ and $K^{(1)}$ as $\delta W=-a_{1} c_{0} \epsilon^{2} / 5$ in the $\alpha \ll 1$ case. Because $c_{0}$ is related to the deformation of spacelike infinity, this term would be related to the work which is necessary for the deformation of infinity. In the next section, we will discuss the origin of this work term in more detail.

Because the work term $\delta W$ in Eq. (93) is negative, the change in the horizon area $\delta A$ of the deformed black hole and that of the Schwarzschild-anti-de Sitter black hole $\delta A_{0}$ for the same $\delta m$ (i.e., $\kappa \delta A_{0} / 8 \pi=\delta m$ ) satisfy the relation $\delta A_{0}<\delta A$. This implies that the area of the deformed black hole is larger than that of the Schwarzschild-anti-de Sitter black hole if compared under the same Ashtekar-Magnon mass. Interestingly, we can claim that the deformation of the Schwarzschild-anti-de Sitter black hole is a process consistent with the area theorem. Although the area theorem has not been proved in the spacetime with $\Lambda<0$ and there are some counterexamples for this theorem such as the black holes in Brans-Dicke 
TABLE I: The value of $\alpha(\delta A / A) /(\delta S / S), \alpha(\delta m / m) /(\delta S / S)$, and $\alpha(\delta W / m) /(\delta S / S)$ for $l=2, \ldots, 9$. The work term is negative for all $l$ and it becomes large as $l$ increases.

\begin{tabular}{c|cccccccc}
\hline \hline$l$ & 2 & 3 & 4 & 5 & 6 & 7 & 8 & 9 \\
\hline$\alpha(\delta A / A) /(\delta S / S)$ & -1.26 & -1.86 & -2.45 & -3.02 & -3.60 & -4.17 & -4.73 & -5.30 \\
$\alpha(\delta m / m) /(\delta S / S)$ & -1.81 & -5.18 & -11.2 & -20.5 & -34.0 & -52.4 & -76.4 & -107. \\
$\alpha(\delta W / m) /(\delta S / S)$ & -1.18 & -4.24 & -9.94 & -19.0 & -32.2 & -50.3 & -74.0 & -104. \\
\hline \hline
\end{tabular}

theory [28], we expect that this result indicates the importance of the solution sequence of the deformed black hole.

Now we discuss the $l>2$ cases. Calculating the integrals in Eqs. (58) and (81) numerically, we evaluate the factor $\alpha(\delta A / A) /(\delta S / S), \alpha(\delta m / m) /(\delta S / S)$, and $\alpha(\delta W / m) /(\delta S / S)$. In all cases, $\delta A, \delta m$, and $\delta W$ are negative. The results are shown in Table I. The work term estimated by $(\delta W / m) /(\delta S / S)$ increases for larger $l$ and the difference from the ordinary thermodynamical relation becomes large. A higher-multipole deformation requires a larger work term. Interestingly, we have found that the relation

$$
\delta W=-\frac{(l-1)(l+2)}{4(2 l+1)} a_{1} c_{0} \epsilon^{2}
$$

holds with accuracy $10^{-6}$. This indicates that this would be the exact value and that our numerical calculation is accurate. Similarly to the $l=2$ case, the area of the deformed black hole is larger than that of the Schwarzschild-anti-de Sitter black hole if compared under the same mass.

\section{B. $\alpha \gg 1$ case}

In the case of $\alpha \gg 1$, we have the relation $x_{h} \simeq \alpha^{-2 / 3} \ll 1$ for the location of the black hole $x=x_{h}$. Near the horizon, the first term of $e^{2 \nu_{0}}=1-1 / x+(\alpha x)^{2}$ is $\alpha^{-2 / 3}$ times smaller compared to the other two terms. In the region $x \gtrsim 1$, the third term $(\alpha x)^{2}$ in $e^{2 \nu_{0}}$ is more than $\alpha^{2}$ times larger than the other two terms. Hence, for all $x_{h} \leq x \leq \infty$, the first term is small compared to the sum of the other two terms and we neglect it in the following analysis. In this approximation, we should neglect terms whose order is $\alpha^{-2 / 3}$ times the leading order.

Setting $z \equiv r / r_{h}$ and $Z \equiv z^{3}$, Eq. (13) is written as

$$
3 Z(Z-1) M_{, Z Z}-(2 Z+1) M_{, Z}+2 M=0,
$$


under the above approximation. The solution satisfying the boundary condition becomes $M=2 Z-3 Z^{2 / 3}+1$, and the corresponding solution $H^{(1)}$ becomes

$$
H^{(1)}=-\frac{1}{z}+\frac{3 z}{z^{2}+z+1}+O\left(\alpha^{-2 / 3}\right) \text {. }
$$

Using Eq. (14), we derive

$$
K^{(1)}=\frac{6 \alpha^{2 / 3}}{l^{2}+l-2}+O(1) .
$$

In summary, the solutions of $H^{(1)}$ and $K^{(1)}$ are

$$
\begin{aligned}
& H^{(1)}=O\left(\alpha^{-2 / 3}\right), \\
& K^{(1)}=(2 l+1)^{1 / 2}+O\left(\alpha^{-2 / 3}\right),
\end{aligned}
$$

where we used the degree of freedom to choose the amplitude of $K^{(1)}$. It is a remarkable fact that $K^{(1)}$ is almost constant and hence $\left|\tilde{c}_{0}\right| \simeq\left|c_{0}\right|$. Although we mentioned in Sec. II that the conformal boundary at infinity is more deformed compared to the horizon, the deformations of the two are similar if $|\Lambda|$ is large. This feature does not depend on $l$. These results are in contrast to the $\alpha \ll 1$ case.

Substituting Eqs. (98) and (99) into Eqs. (58), (59), and (81), we immediately find

$$
\begin{aligned}
\delta A / A & =\left[2+O\left(\alpha^{-2 / 3}\right)\right] \epsilon^{2}, \\
\delta S / S & =\left[2+O\left(\alpha^{-2 / 3}\right)\right] \epsilon^{2}, \\
\delta m / m & =\left[3+O\left(\alpha^{-2 / 3}\right)\right] \epsilon^{2} .
\end{aligned}
$$

In this case, the back reaction to the horizon area is similar to that to the spacelike area at infinity. This result leads to

$$
\delta m \simeq(\kappa / 8 \pi) \delta A
$$

which means that the thermodynamic law of the deformed black holes is almost the same as that of the Schwarzschild-anti-de Sitter black holes if $|\Lambda|$ is sufficiently large. In contrast to the $\alpha \ll 1$ case, the work term is small in the $\alpha \gg 1$ case. Hence we find that there is a correlation between the value of the work term and the difference of the deformation of the horizon and the spacelike surface at infinity: the absolute value of the work term decreases as $\left|\tilde{c}_{0} / c_{0}\right|$ approaches unity. 


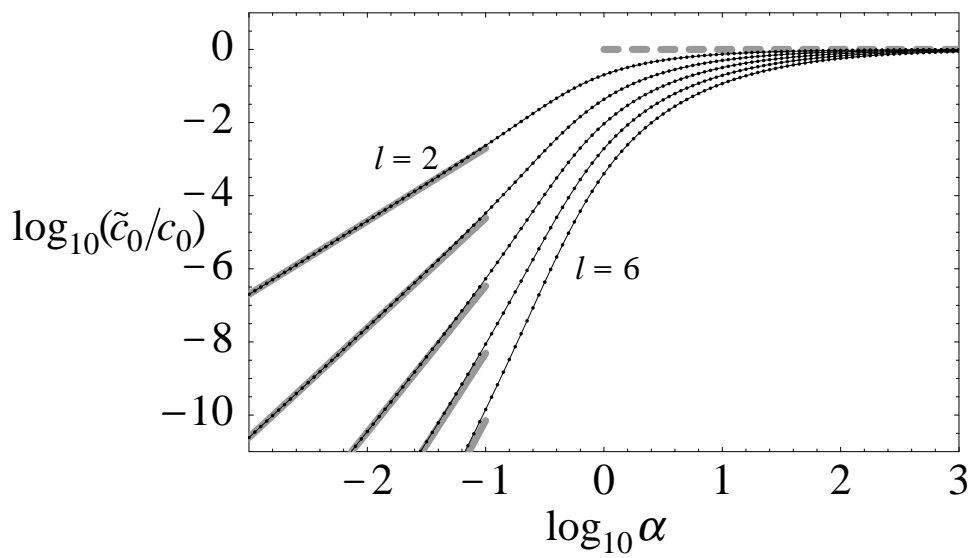

FIG. 1: The relation of $\alpha$ and the ratio of the deformation of the horizon and spacelike infinity $\tilde{c}_{0} / c_{0}$ for $l=2, \ldots, 6$. Both axes are shown in the log scale. The gray dashed line shows the asymptotic behavior for $\alpha \gg 1$ and the gray solid line shows the asymptotic behavior for $\alpha \ll 1$ for each $l$.

\section{NUMERICAL CALCULATION FOR $\alpha \sim 1$}

In this section, we numerically investigate the deformed black hole for $\alpha \sim 1$ to complete the perturbative analysis. Equations (91), (10), (11), and (12) can be reduced to two firstorder differential equations for $\left(r H^{(1)}\right)$ and $K^{(1)}$, which asymptote to constant values for large $r$. We solved these equations using the Runge-Kutta method from the horizon $r=r_{h}$ to the cutoff value $r=r_{c}$. We selected the grid number and the cutoff value $r=r_{c}$ as follows. Because there are two characteristic length scales $R_{A}$ and $R_{S}$, we set $10^{2}$ grids within the smaller length scale and solved in a range which is $10^{2}$ times as long as the larger length scale. Hence, the cutoff value is $r_{c}=r_{h}+10^{2} \times \max \left[R_{A}, R_{S}\right]$ and the total grid number becomes $10^{4} \times \max \left[\alpha, \alpha^{-1}\right]$. Beyond the cutoff $r=r_{c}$, we approximate $H^{(1)}$ and $K^{(1)}$ with the formulas $H^{(1)} \simeq a_{1} / r+a_{2} / r^{2}$ and $K^{(1)} \simeq c_{0}+c_{1} / r$, from which we determine the value of $c_{0}$ and $a_{1}$ using Eq. (19), Eq. (20), and the numerical values of $\left(r H^{(1)}\right)$ and $K^{(1)}$ at $r=r_{c}$, and evaluate the integrals in Eqs. (58) and (81) beyond the cutoff $r>r_{c}$. The numerical error is about $0.1 \%$, which is estimated by using several different grid numbers and the cutoff values. In the $\alpha=10^{-3}$ case, our numerical results coincide with the values in Table I with $0.1 \%$ accuracy.

Now we show the numerical results. Figure 1 shows the behavior of the ratio $\left(\tilde{c}_{0} / c_{0}\right)$ of the deformation of the two spacetime boundaries - i.e., the horizon and the two-surface at spacelike infinity - as a function of $\alpha$. The asymptotic behaviors for $\alpha \gg 1$ and $\alpha \ll 1$ derived in 


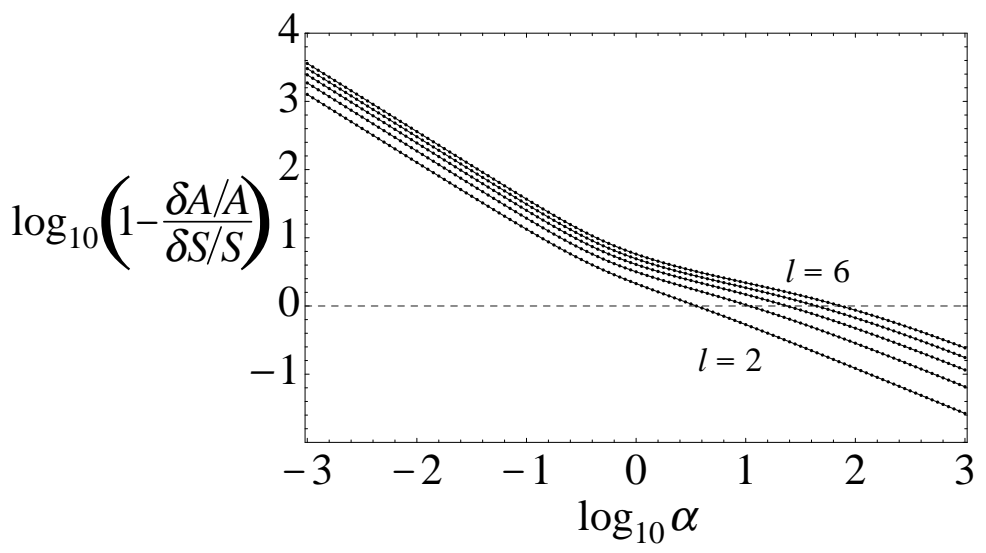

FIG. 2: The relation of $\alpha$ and change in the horizon area $1-(\delta A / A) /(\delta S / S)$ for $l=2, \ldots, 6$. Both axes are shown in the $\log$ scale. The value of $1-(\delta A / A) /(\delta S / S)$ is almost proportional to $\alpha^{-1}$ for $\alpha \ll 1$ and is proportional to $\alpha^{-2 / 3}$ for $\alpha \gg 1$. The location where $\delta A$ becomes zero is shown by a dashed line.

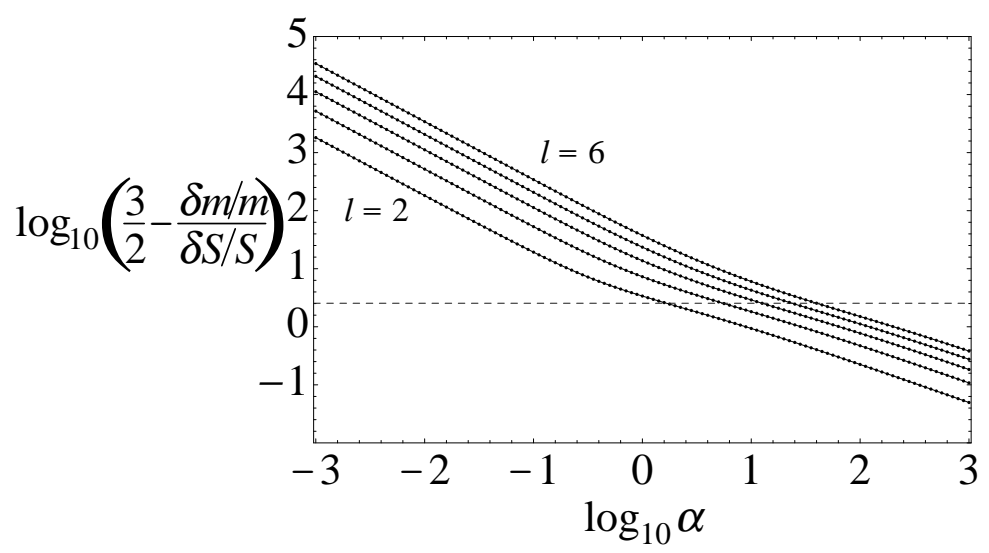

FIG. 3: The relation of $\alpha$ and the change in the mass $3 / 2-(\delta m / m) /(\delta S / S)$ for $l=2, \ldots, 6$. Both axes are shown in the $\log$ scale. The value of $3 / 2-(\delta m / m) /(\delta S / S)$ is almost proportional to $\alpha^{-1}$ for $\alpha \ll 1$ and is proportional to $\alpha^{-2 / 3}$ for $\alpha \gg 1$. The location where $\delta m$ becomes zero is shown by a dashed line.

Sec. IV are also shown. We see that the matching method gives a fairy good approximation for $\alpha \lesssim 10^{-1}$. The behavior of $1-(\delta A / A) /(\delta S / S)$ and $3 / 2-(\delta m / m) /(\delta S / S)$ are shown in Figs. 2 and 3, respectively. We see that both $(\delta A / A) /(\delta S / S)$ and $(\delta m / m) /(\delta S / S)$ are proportional to $\alpha^{-1}$ for $\alpha \ll 1$, which is consistent with the analysis of Sec. IV. In the region $\alpha \gg 1,1-(\delta A / A) /(\delta S / S)$ and $3 / 2-(\delta m / m) /(\delta S / S)$ asymptote to zero and these values are proportional to $\alpha^{-2 / 3}$. This is also consistent with the results in Sec. IV. 


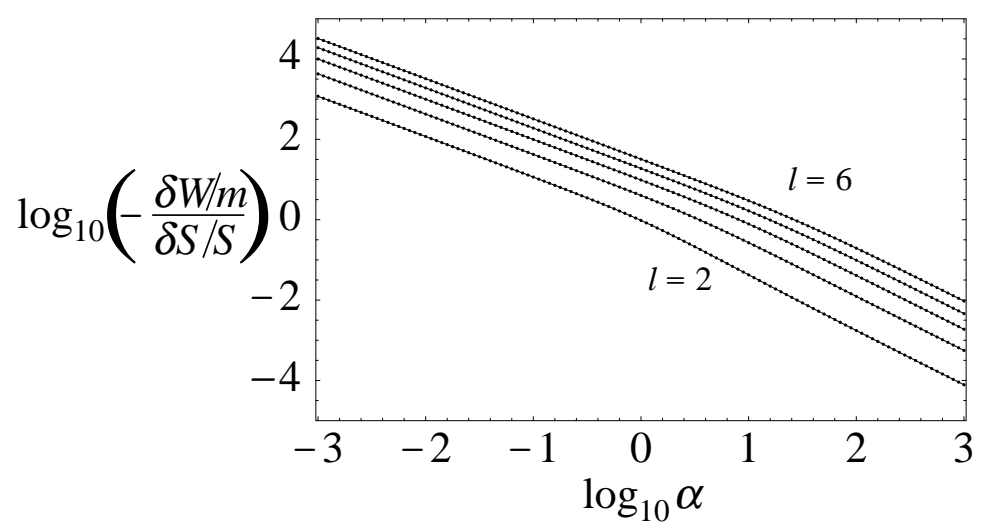

FIG. 4: The relation of $\alpha$ and the work term $-(\delta W / m) /(\delta S / S)$ for $l=2, \ldots, 6$. Both axis are shown in the log scale. The value of $-(\delta W / m) /(\delta S / S)$ is almost proportional to $\alpha^{-1}$ for $\alpha \ll 1$ and is proportional to $\alpha^{-4 / 3}$ for $\alpha \gg 1$.

Figure 4 shows the behavior of the work term $(\delta W / m) /(\delta S / S)$. We see that the value of $\log _{10}[-(\delta W / m) /(\delta S / S)]$ does not diverge and hence $\delta W$ always takes a negative value. This implies that the area of the deformed black hole is larger than that of the Schwarzschild-antide Sitter black hole if compared under the same Ashtekar-Magnon mass for arbitrary $\alpha$. In the region $\alpha \ll 1$, the value of $(\delta W / m) /(\delta S / S)$ is proportional to $\alpha^{-1}$, which coincides with the results of Sec. IV. This behavior of $(\delta W / m) /(\delta S / S)$ indicates that quasistatic deformation requires a larger absolute value of the work for smaller $\alpha$ and it is consistent with the fact that the Schwarzschild spacetime does not allow the quasistatic deformation. The value of $(\delta W / m) /(\delta S / S)$ is proportional to $\alpha^{-4 / 3}$ in the $\alpha \gg 1$ region, although both $1-(\delta A / A) /(\delta S / S)$ and $3 / 2-(\delta m / m) /(\delta S / S)$ are proportional to $\alpha^{-2 / 3}$. This is probably because the terms which are proportional to $\alpha^{-2 / 3}$ in $\delta A / A$ and $\delta m / m$ cancel each other in the calculation of the work term, although we have not proceeded this analysis. The work term rapidly decreases in the region $\alpha \gg 1$. For all $\alpha$, the absolute value of $(\delta W / m) /(\delta S / S)$ becomes large as $l$ increases. The higher-multipole deformation requires a larger work term.

Figure 5 shows the value of $\delta W / a_{1} c_{0} \epsilon^{2}$, which was introduced with Eq. (94) in the approximate analysis for $\alpha \ll 1$ in Sec. IV. The value of $\delta W / a_{1} c_{0} \epsilon^{2}$ is almost constant in the region where the matching method provides a good approximation. Although this quantity slightly changes with an increase of $\alpha$, it seems to asymptote to some nonzero constant value. This is supported by the following consideration. For $\alpha \gg 1$, we see that $K^{(1)} \sim 1$ and $H^{(1)} \sim \alpha^{-2 / 3} / z$ from Eqs. (98) and (99). Using $z=r / r_{h}$ and $r_{h} \simeq 2 m \alpha^{-2 / 3}$, we have 


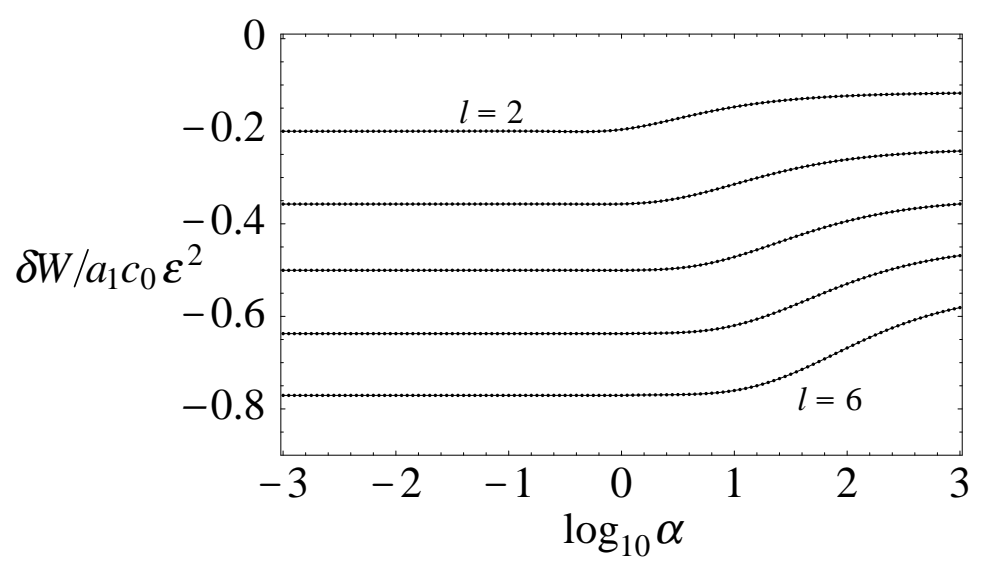

FIG. 5: The relation of $\alpha$ and the value of $\delta W / a_{1} c_{0} \epsilon^{2}$ for $l=2, \ldots, 6$. Here $\delta W / a_{1} c_{0} \epsilon^{2}$ is almost constant for $\alpha \lesssim 1$. If we increase $\alpha, \delta W / a_{1} c_{0} \epsilon^{2}$ slightly changes and asymptotes to some nonzero constant value.

$a_{1} \sim \alpha^{-4 / 3} m$ and thus $a_{1} c_{0} \epsilon^{2} \sim \alpha^{-4 / 3} m \epsilon^{2}$. This leads to $-a_{1} c_{0} \epsilon^{2} \sim \delta W$ for $\alpha \gg 1$. Hence the value of $-\delta W / a_{1} c_{0} \epsilon^{2}$ is always $O(1)$ and the relation, Eq. (94), approximately holds for all $\alpha$.

We can construct another quantity which has the same order as $\delta W$ as follows:

$$
-\frac{\delta W}{r_{h}} \sim\left(1-\frac{r_{h}}{2 m}\right)^{-1 / 2}\left(c_{0}^{2}-\tilde{c}_{0}^{2}\right)
$$

According to this formula, the work term normalized by the horizon radius depends only on two factors. One is related to the horizon radius, which shows that the absolute value of the work term becomes larger for smaller $\alpha$. The other factor depends on the values of $\tilde{c}_{0}$ and $c_{0}$, which are related to the deformation of the horizon and spacelike infinity, respectively. Because the deformation of the two boundary surfaces becomes similar $\left|\tilde{c}_{0} / c_{0}\right| \simeq 1$ for larger $\alpha$, the absolute value of the work term decreases with an increase of $\alpha$. Conversely, it becomes large with a decrease in $\alpha$, which corresponds to a decrease in $\left|\tilde{c}_{0} / c_{0}\right|$. Hence we interpret that the origin of the work term is related to the difference of the deformation between the two boundary surfaces of the spacetime. The work would be necessary for the deformation of both horizon and spacelike infinity, and their difference would appear in the first law as the work term. 


\section{SUMMARY AND DISCUSSION}

In this paper, we analyzed the static deformation of the Schwarzschild-anti-de Sitter black holes using perturbative techniques. We showed that there exists a regular solution for the first-order perturbation. The resulting spacetime contains the deformed black hole whose horizon deviates from the geometrically spherically symmetric surface. The spacelike infinity of this spacetime is deformed simultaneously. Hence this spacetime is not asymptotically anti-de Sitter, although this is still weakly asymptotically anti-de Sitter in the sense of the Ashtekar-Magnon definition [20]. In the $\alpha \ll 1$ case, the deformation of the horizon is about $\alpha^{l}$ times smaller than the deformation of spacelike infinity, while the deformations of the two are similar in the $\alpha \gg 1$ case.

We considered the $l=0$ mode of the second-order perturbation and calculated the change in the horizon area and the mass. In the mass calculation, we used two definitions of the mass: the Abbott-Deser mass and the Ashtekar-Magnon mass. The Abbott-Deser mass for the deformed black hole diverges to minus infinity. If this result is realistic, we are forced to conclude that the Schwarzschild-anti-de Sitter black hole would rapidly deform toward the lower-energy state. But this definition is not gauge invariant at second order and we consider that this result may be spurious. Because the Ashtekar-Magnon mass is a covariant definition of the mass from which we obtained finite results, we expect that it represents a real amount of energy although one assumption is imposed in Eq. (75) in choosing the norm of $\xi^{t}$. Our results indicate that the quasistatic deformation of the spacetime boundaries may occur with a finite change in the total energy in the weakly asymptotically anti-de Sitter spacetimes. Of course only with the analysis in this paper, we cannot rigorously conclude that such a process actually occurs. But our expectation that the spacetime boundaries are flexible is also supported by the fact that no one has proved that spacelike infinity of the weakly asymptotically anti-de Sitter spacetime should be rigid; i.e., it has a global timelike conformal Killing vector field. There might exist many weakly asymptotically anti-de Sitter solutions whose geometrical configuration at spacelike infinity temporally evolves.

We studied the thermodynamic first law of the deformed black holes using the AshtekarMagnon mass. In the $\alpha \gg 1$ case, the first law in the usual form is approximately recovered, while in the $\alpha \ll 1$ case, the first law does not hold: the contribution of the work term $\delta W$ becomes important. In Sec. $\mathrm{V}$, we numerically calculated this work term in the range 
$10^{-3} \leq \alpha \leq 10^{3}$ for $l=2, \ldots, 6$ and confirmed that it is always negative in this regime. Let us discuss the implications of this first law from the viewpoint of black hole thermodynamics. Although the ratio of $\delta m / m$ and $\delta A / A$ is fixed for each $\alpha$ in Secs. IV and $\mathrm{V}$, we can consider these parameters to be independent if we further take account of the thermodynamical relation of the background Schwarzschild-anti-de Sitter spacetime, $\delta m_{0}=(\kappa / 8 \pi) \delta A_{0}$. The first law for the deformation process is given by $\delta m=(\kappa / 8 \pi) \delta A+\delta W$ with $\delta W<0$ for two independent parameters $\delta m$ and $\delta A$. If the deformation process occurs without changing the horizon area (i.e., $\delta A=0$ and $\delta m=\delta W$ ), the black hole mass decreases (i.e., $\delta m<0$ ) and the black hole evolves towards the lower-energy state. Hence the deformation may work as a process of energy extraction from a spherical black hole. On the other hand, if there is a deformation process in which the black hole mass does not change, the negative work term implies that the horizon area of the deformed black hole increases in this process. This indicates that quasistatic deformation can be a process consistent with the area theorem. Although whether the area theorem holds for these spacetimes is quite uncertain, we expect that this is an indication for the importance of the solution series of the deformed black holes. The value of $(\delta W / m) /(\delta S / S)$ is given in Fig. 4 as a function of $\alpha$. It is proportional to $\alpha^{-1}$ for $\alpha \ll 1$. This indicates that the deformation requires the larger absolute value of the work for smaller $\alpha$. This is consistent with the fact that the Schwarzschild spacetime does not allow a continuous static deformation. For $\alpha \gg 1$, the absolute value of $(\delta W / m) /(\delta S / S)$ is proportional to $\alpha^{-4 / 3}$ and rapidly decreases with the increase in $\alpha$. The higher-multipole deformation requires a larger absolute value of the work term. Because the work term satisfies the relation (104) for any $\alpha$, this term is closely related to the difference of the deformation between the two boundaries of this spacetime: i.e., the horizon and spacelike two-surface at infinity.

Here, we discuss the reason why the work term is negative using the Hartle-Hawking formula 29] (see also [30] for a review). Using the Raychaudhuri equation, Hartle and Hawking derived the following formula for an increase in the horizon area in the quasistationary evolution of a black hole:

$$
\frac{\kappa}{8 \pi} \delta A=\oint d A \int_{t_{0}}^{t_{1}}\left(\frac{\sigma^{2}}{16 \pi}+T_{a b} l^{a} l^{b}\right) d t,
$$

where $t_{0}$ and $t_{1}$ denote the time for the initial state and final state, respectively, $\sigma$ denotes the shear scalar of the null geodesic congruence of the horizon, $T_{a b}$ is the energy-momentum 
tensor of the matter field that crosses the horizon, and $l^{a}$ is the Killing vector field on the horizon. In the asymptotically flat case, this formula is equivalent to the thermodynamic first law. If matter crosses the horizon and the flow of gravitational wave energy can be ignored, the second term of the integral in Eq. (105) becomes the change of mass $\delta M$ and the first term becomes zero because $\sigma$ has the same order as $\delta A$ and thus $\sigma^{2}$ is much smaller than $\delta A$. (Here we do not consider any change of angular momentum.) If there is no matter and the gravitational wave energy is absorbed into the black hole, the first term has the same order as $\delta A$ in this case and gives $\delta M$. Hence the first law can be derived in general. The first term of the integral in Eq. (105) has an analogy with the entropy generated by a surface shear viscosity with magnitude $\eta_{\nu}=1 / 16 \pi$ of the ordinary viscous fluid. Now we discuss the application of this Hartle-Hawking formula to the deformed black hole. If some energy flux crosses the Schwarzschild-anti-de Sitter horizon to induce a quasistatic deformation of the spacetime, a part of the first term of the integral in Eq. (105) would contribute to the work term $\delta W$ necessary for the deformation, while the remaining part (in addition to the second term) would contribute to the mass term $\delta M$. The important fact is that the shear $\sigma$ is $O(\epsilon)$ in this case, and hence all $\delta A, \delta M$, and $\sigma^{2}$ have the same order $O\left(\epsilon^{2}\right)$. The first term of the integral in Eq. (105) cannot be ignored, and introducing an unknown positive parameter $\beta$, the work term and mass term may be given by

$$
\begin{aligned}
& \delta W=-\oint d A \int_{t_{0}}^{t_{1}}\left(\beta \sigma^{2} / 16 \pi\right) d t, \\
& \delta M=\oint d A \int_{t_{0}}^{t_{1}}\left(\frac{(1-\beta) \sigma^{2}}{16 \pi}+T_{a b} l^{a} l^{b}\right) d t,
\end{aligned}
$$

which requires the work term to be negative. Although we have not investigated the validity of the relations in terms of the Ashtekar-Magnon mass, this discussion provides a natural interpretation of the reason why the work term obtained in this paper becomes negative.

Our remaining problems are as follows. As for the problems of the solution sequence of deformed black holes, we should construct solutions beyond the perturbative region. This would probably require numerical calculations. Because spacelike infinity is also deformed, we should impose the structure of spacelike infinity in the calculation. This arbitrariness in choosing the infinity structure would lead to great degrees of freedom of the solution series of deformed black holes. The condition for the existence of a solution due to the choice of infinity structure is of interest. Next, we should analyze the stability of spacetimes with deformed black holes. This will require an analysis of the quasinormal frequency of the 
deformed black holes. At the same time, we would like to analyze the perturbation from the Schwarzschild-anti-de Sitter black hole which gives the time-dependent geometrical configuration at spacelike infinity. With this analysis, what spacetime is an attractor of the weakly asymptotically anti-de Sitter spacetimes might become clear. Concerning the application of these spacetimes with deformed black holes, these solutions might contribute to the brane world scenario. Although our analysis is restricted to four-dimensional cases, it is natural to expect that similar solutions exist in higher dimensions. Hence, by appropriate cutting and pasting, these spacetimes might provide interesting models of the brane world scenario. Finally, we would like to analyze the implication for the AdS/CFT correspondence of these solutions. In the usual Schwarzschild-anti-de Sitter black holes, the quasinormal frequencies have a relation to the correlation function of the field on the boundary. By analyzing this correspondence in the spacetime with deformed black holes, these spacetimes might shed new light on the AdS/CFT correspondence. These are the problems we would like to investigate as the next step.

\section{Acknowledgments}

The work of H.Y. is supported in part by a grant-in-aid from Nagoya University 21st Century COE Program (ORIUM).

[1] O. Aharony, S. S. Gubser, J. Maldacena, H. Ooguri, and Y. Oz, Phys. Rep. 323, 183 (2000).

[2] L. Randall and R. Sundrum, Phys. Rev. Lett. 83, 3370 (1999); 83, 4690 (1999).

[3] J. P. S. Lemos, Class. Quantum Grav. 12, 1081 (1995).

[4] J. P. S. Lemos, Phys. Lett. B 353, 46 (1995).

[5] C.-g. Huang and C.-b. Liang, Phys. Lett. A 201, 27 (1995).

[6] J. P. S. Lemos and V. T. Zanchin, Phys. Rev. D 54, 3840 (1996).

[7] R. G. Cai and Y. Z. Zhang, Phys. Rev. D 54, 4891 (1996).

[8] S. Åminneborg, I. Bengtsson, S. Holst, and P. Peldán, Class. Quantum Grav. 13, 2707 (1996).

[9] D. R. Brill, Helv. Phys. Acta 69, 249 (1996).

[10] R. B. Mann, Class. Quantum Grav. 14, L109 (1997). 
[11] D. Klemm, V. Moretti, and L. Vanzo, Phys. Rev. D 57, 6127 (1998); 60, 109902(E) (1999).

[12] S. W. Hawking, Commun. Math. Phys. 25, 152 (1972).

[13] M. Anderson, P. T. Chruściel, and E. Delay, J. High Energy Phys. 10, 063 (2002).

[14] G. J. Galloway, S. Surya, and E. Woolgar, Class. Quantum Grav. 20, 1635 (2003).

[15] R. Gregory and R. Laflamme, Phys. Rev. Lett. 70, 2837 (1993).

[16] S. S. Gubser, Class. Quantum Grav. 19, 4825 (2002).

[17] T. Wiseman, Class. Quantum Grav. 20, 1137 (2003).

[18] H. Kudoh and T. Wiseman, Prog. Theor. Phys. 111, 475 (2004).

[19] T. Regge and J. A. Wheeler, Phys. Rev. 108, 1063 (1957).

[20] A. Ashtekar and A. Magnon, Class. Quantum Grav. 1, L39 (1984).

[21] P. T. Chruściel and G. Nagy, Adv. Theor. Math. Phys. 5, 697 (2002).

[22] L. F. Abbott and S. Deser, Nucl. Phys. B195, 76 (1982).

[23] L. Vanzo, Phys. Rev. D 56, 6475 (1997).

[24] M. M. Caldarelli, G. Cognola, and D. Klemm, Class. Quantum Grav. 17, 399 (2000).

[25] S. Silva, Class. Quantum Grav. 19, 3947 (2002).

[26] G. Barnich, Class. Quantum Grav. 20, 3685 (2003).

[27] R. J. Gleiser, C. O. Nicasio, R. H. Price, and J. Pullin, Phys. Rep. 325, 41 (2000).

[28] G. Kang, Phys. Rev. D 54, 7483 (1996).

[29] J. B. Hartle and S. W. Hawking, Commun. Math. Phys. 27, 283 (1972).

[30] B. Carter, in General Relativity, edited by S. W. Hawking and W. Israel (Cambridge University Press, Chambridge, England, 1979). 